\title{
Investigation of a Linear Optical Fiber Sensing and Monitoring System
}

\author{
Jian Chen ${ }^{1, a}$, Wei li Bai ${ }^{2, b}$ \\ ${ }^{1}$ School of Electro-Mechanical Engineering, Guangdong University of Technology, Guangzhou \\ 510006, Guangdong, China \\ ${ }^{2}$ Department of Electronics, Guangzhou Vocational College of Science and Technology, Guangzhou \\ 510550, Guangdong, China \\ achenjian@gdut.edu.cn, ${ }^{b} 1036685924 @ q q . c o m$
}

Keywords: distributed fiber, Raman-scattering, Qt, fire detection, MySQL database

Abstract. In this paper, monitoring software based on principle and structure of distributed optical fiber fire detection used to observe the temperature of the cable laying and realize the real-time alarm is designed. It was written in Qt Programming language with cooperation of optimization design of the signal acquisition hardware system. This system can monitor and display the spatial temperature distribution of the fiber optic fiber accurately, and has the characteristics of friendly interface, strong real-time performance, etc..

\section{Introduction}

In recent years, more and more large underground buildings such as supermarket, subway tunnels, underground parking lot are built or being built. Because the fire of these underground buildings spreads quickly and results in a large smoke toxicity, personnel evacuation and fire control become a big problem in case of fire, and there will often be a huge loss of property and casualties. So, early detection and warning of fire is particularly important for firefighting [1].

The distributed optical fiber temperature sensing technology is a real time, on-line, multi point temperature sensing technology developed in recent decades and proposed by the University of Southampton in 1981. At present, the temperature can be measured in the range of tens of kilometers [2]. The distributed optical fiber sensing system has the ability of sensing and transmitting of signals and the ability of detecting the physical parameters of the measured surface along the optical fiber position [3]. However, the Raman backscattering light which contains the detected information of temperature is very weak and requires fast acquisition speed. A high speed data acquisition system based on high speed ADC, large scale FPGA and high performance DSP is proposed in this paper, and the input signal is amplified by the LM382 amplifier chip. A distributed optical fiber temperature sensing fire detection and monitoring system based on Qt programming language is designed. The temperature data is sent to the upper computer in real time, and is displayed. So, the real-time monitoring of fire and fire alarming which could greatly improve the safety and efficiency of operation of the fire monitoring system is realized.

\section{Principle of Distributed Fiber Temperature Sensing System}

\section{Basic technical principle}

The distributed optical fiber temperature sensing fire detection system is based on optical time domain reflection technology (OTDR) and Raman scattering temperature effect [4]. A narrow beam of laser pulse is transmitted to the temperature sensing optical fiber through a bidirectional coupler, and a back scattered light generated in the optical fiber is received by the photoelectric detector through the bidirectional coupler [5], as shown in Fig.1. The time T between transmitting and receiving of laser and the distance $L$ passed optical pulses detected by optical fibers are satisfied with the equation:

$$
\mathrm{L}=\mathrm{C}\left|\frac{\mathrm{T}}{2}\right| n,
$$


Where $\mathrm{C}$ is the speed of light, $\mathrm{n}$ is refractive index of light, the time interval $\mathrm{T}$ is corresponding to the optical fiber distance L. Therefore, with the change of $\mathrm{L}$, the detector can measure the spatial distribution along the optical fiber distribution.

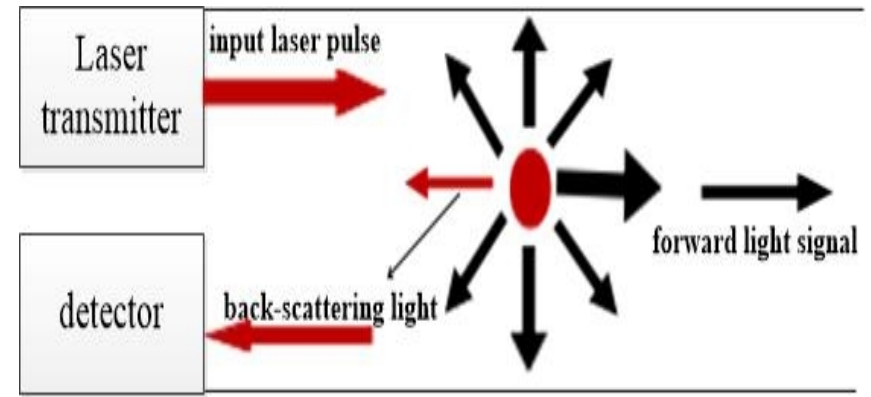

Fig.1 Principle of optical time domain reflection structure diagram

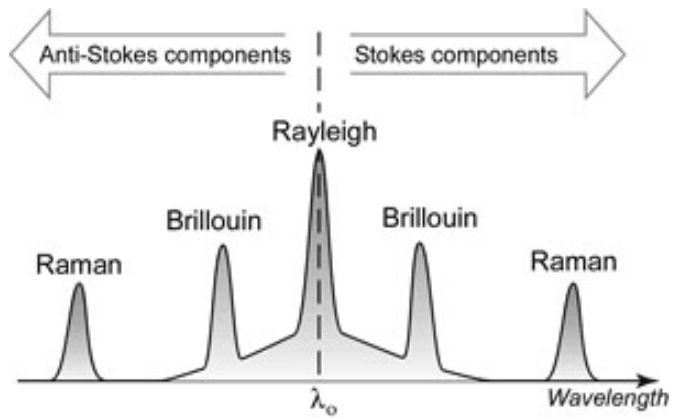

Fig.2 Scattered light intensity wavelength distribution

When the temperature measuring host sends the laser pulse with some energy and width, and the light shines on the light particles, light pulse react with optical fiber molecules and generate scattering. There are many kinds of scattering light, as shown in Fig.2. The thermal vibration of the optical fiber molecule caused the Raman scattering, and produced the Stokes and anti-Stokes light. The temperature can be measured by the ratio of anti-Stokes light intensity to Stokes light intensity:

$$
R(T)=\frac{I_{a s}(T)}{I_{s}}=\left(\frac{\lambda_{s}}{\lambda_{a s}}\right)^{4} \exp \left(-\frac{h c v_{0}}{k T}\right),
$$

Where ratio $R(T)$ is only related to temperature. So we can get the temperature value of the detected optical fiber by measuring the ratio of the anti-Stokes light to Stokes light intensity, and then obtain the spatial temperature field distribution by the optical time domain reflection technique (OTDR).

The structure of the system

The basic structure of the detection system is shown in Fig.3. The system is mainly composed of driving circuit, laser transmitter, wavelength division multiplexer, temperature sensing optical fiber, amplifier circuit, signal acquisition and processing part and computer system.
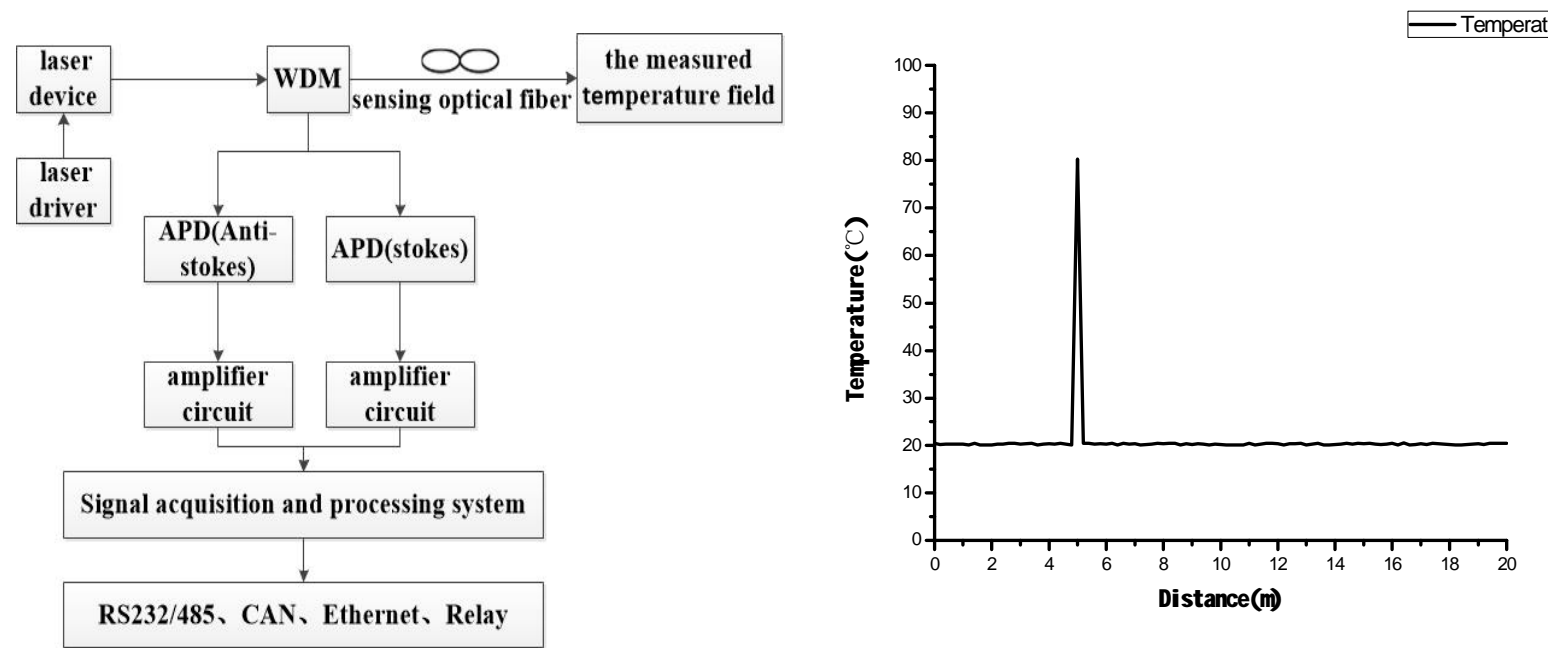

Fig. 3 block diagram of distributed optical Fig.4 curve of temperature versus distance distribution The power drives the laser to transmit a laser pulse, and then is coupled to the multimode data optical fiber through a wavelength division multiplexer (WDM), simultaneously drives the data acquisition board to realize the synchronous signal acquisition [6]. The temperature sensing optical fiber to be measured acquires temperature information, and laser pulse reacts with some of the uneven distribution of particles within the fiber to produce scattering phenomenal in which the scattering light propagates along the fiber in the opposite direction. Once again it enters the wavelength division multiplexer, the anti-Stokes light and Stokes light were extracted separately, and the other scattered light was suppressed. And then through the coupler into the avalanche photodiode APD, the optical 
signal was changed into electrical signals. After subsequent amplification, the temperature data collected through the DSP digital signal processing and then sent to the computer to display. According to the time difference between the transmission rate of the optical fiber and the back light echo, and the accurate positioning of the measured temperature point by the OTDR technology, the temperature value and the changing trend of each point are displayed through the computer.

\section{The PC HMI Monitoring Software Design}

The alarm extension equipment transfers real-time temperature and control information to the upper PC computer through RS232. The PC computer is based on Windows operating system, HMI software is developed using the Qt object-oriented programming language and MySQL database. In order to display information of the distribution of the optical fiber in real time and to realize the function of temperature-based fire warning, the Human Machine Interface software has the following functions:

\section{Accurate detection}

The HMI software can clearly display the specific location and code number of the laying optical fiber, can realize real-time online temperature monitoring and fire alarming, and can obtain accurate location information. In this display area, the user can see the temperature of the whole laying optical fiber at a certain time point along with the distance distribution. Fig. 4 shows the curve of temperature versus distance distribution. In the place about 5 meters, the temperature reaches 80 degrees Celsius, which is abnormal, and an alarm should be triggered.

\section{Electronic map orientation}

The electronic map can display actual laying direction and position of temperature sensing optical fiber and temperature information along the whole or partial optical fiber length. It can also provide real-time data of ambient temperature between different segments and groups, alarm information, and fire spread direction etc. The design of electronic map module includes group, segment and curve, which are called different alarm objects, as shown in Fig.5.

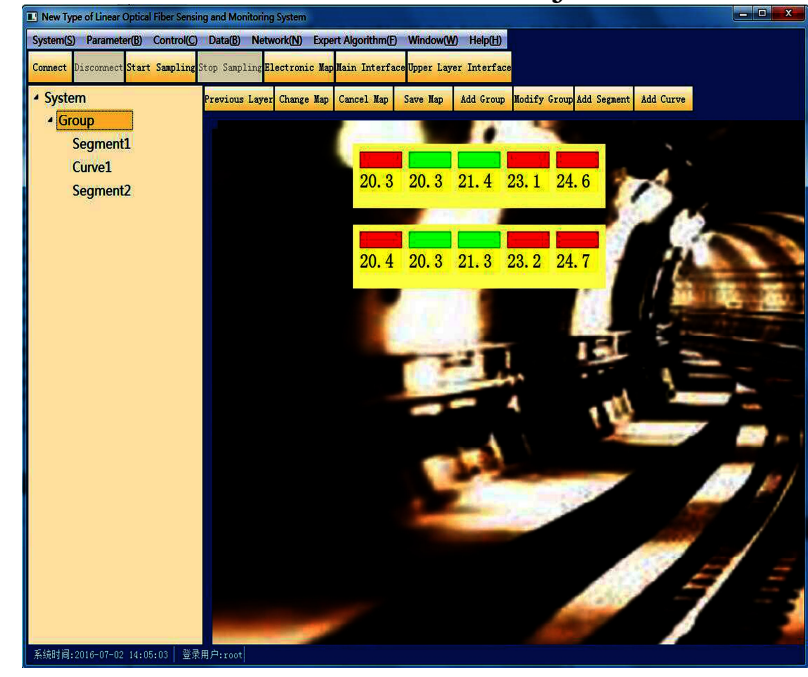

Fig.5 electronic map design interface

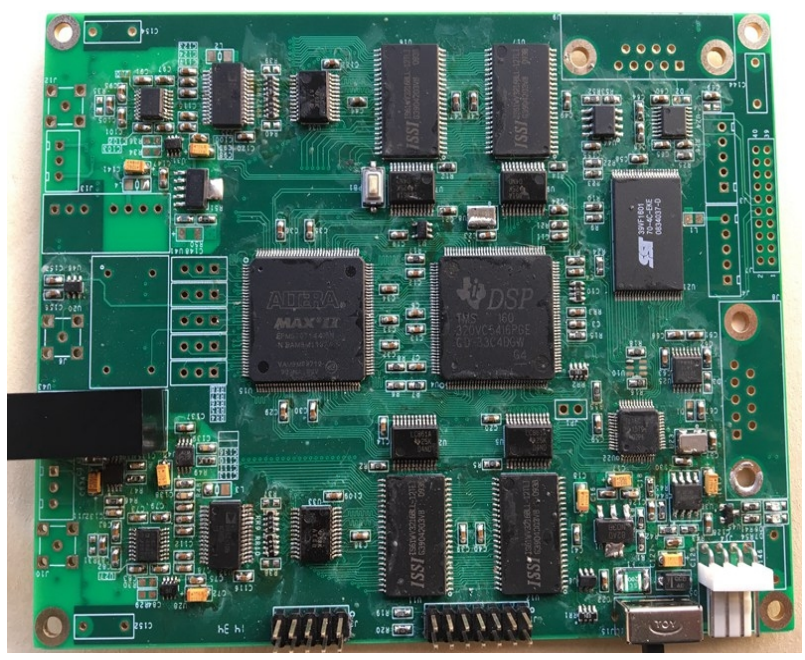

Fig.6 high speed data acquisition board

\section{Alarm settings}

The HMI software also has the function of automatic alarm that can be set to various alarm level including first level warning, second level warning, fire warning, optical fiber failure warning, system failure warning, temperature rising warning and other warning level.

Historical records

All the original sampling data is stored in the MySQL database. User can query temperature record and alarm record that has happened. As long as the user gives the query conditions, such as time, alarm type, connection number, cable section, etc., reports will be generated automatically. By calling the API function to establish a MySQL database connection, we need first to put the database drive library MySQL libmysql.dll under the current directory. 
1. Enable database driver

QSqlDatabase $\mathrm{db}=$ QSqlDatabase::addDatabase("QMYSQL", sName);

Where the second parameter is the database connection name.

2. Open database

Call db.open() to open database connection.

\section{Hardware System}

According to the distributed optical fiber parameters, measurement accuracy, spatial resolution, measurement time and distance, etc., combined with the special requirements of fire detection, the hardware system needs to have a strong, real-time data acquisition and processing capabilities. The high-speed AD9814, large scale FPGA and high performance digital signal processing unit TMS320VC5416 are used, as shown in Fig.6.

\section{Conclusions}

Real time sampling of high speed signal for the distributed optical fiber temperature sensing detection system and Qt-based Human Machine Interface are realized. The monitoring method is very flexible. It can monitor not only temperature of the whole optical fiber, but also detailed distribution of the whole optical fiber. The temperature detection ranging from $-30{ }^{\circ} \mathrm{C} \sim 120{ }^{\circ} \mathrm{C}$ and the measuring distance up to several kilometers long are achieved. The temperature measurement accuracy and the positioning accuracy are up to $+1{ }^{\circ} \mathrm{C}$ and $1 \mathrm{~m}$ respectively, and the temperature state at any position can be displayed.

\section{Acknowledgements}

This work was financially supported by Guangdong Provincial Science and Technology Plan Project (2014A010103027), and Huizhou Municipal Science and Technology Plan Project (2015B020005007).

\section{References}

[1] Sumin Liu, Hui Liu, Bin Yao, et al: Application of Optical Fiber Grating Temperature Detection System in Metro Tunnel. Urban Mass Transit [J] (In Chinese), 2012(9):49-52.

[2] Mengmeng Jiang: Research on Several Influence Factors in the Road Tunnel Application of Distributed Optical Fiber Temperature Fire Detection System [D] (In Chinese). Hefei: University of Science and Technology of China, 2014.

[3] Wenhai Sun, Bingbin Li: Application Research of Distributed Optical Fiber Sensing System for Fire Detection and Alarm System. Fire Technique and Products Information [J] (In Chinese), 2014(5):14-17.

[4] Man Fang: Research on Temperature Sensor System and Temperature Resolution Enhancement of Distributed Raman Optical Fiber [D] (In Chinese). Chengdu: University of Electronic Science and Technology, 2004.

[5] Abhisek Ukil, Hubert Braendle, Peter Krippner: Distributed Temperature Sensing: Review of Technology and Applications. IEEE Sensors Journal, Vol. 12, No. 5, May 2012.

[6] Lei Sang, Jie Zhang, Xinqun Wang: Application of Distributed Optical Fiber Temperature Sensing Fire Detection System in one Subway Tunnel [J] (In Chinese). Industrial Safety and Environmental Protection, 2013, 39 (8):52-55. 